\title{
Subretinal Gas
}

\author{
D. WONG*, A. M. ANSONS*, A. H. CHIGNELL*, R. YEOH \\ Liverpool and London
}

\begin{abstract}
Summary
Two cases of subretinal gas are described. The circumstances in which this complication arose are important in understanding the mechanism by which gas gains access into the subretinal space. Evacuation of subretinal gas by posturing should be avoided as this leads to an increase in the extent of the retinal detachment and its extension anterior to involve the nonpigment epithelium of the pars plana. A technique for removing subretinal bubbles of expanding gases using vitrectomy and fluid/gas exchange is recommended.
\end{abstract}

Subretinal gas is an uncommon and severe complication of surgery for retinal detachment, rendering reattachment much more difficult. In 1968, Chawla listed the passage of air under the retina as one of the possible complications for the use of air, although this did not occur in any of the cases presented in his paper. ${ }^{1}$ In 1986 , Lincoff described a single case in pseudophakic retinal detachment, ${ }^{2}$ and in 1987 McDonald et al., and in 1988 Lowe et al., reported eight cases of subretinal gas following pneumatic retinopexy.,4

The mechanism by which gas gained access into the subretinal space has not been fully explained. In the one case described by Lincoff, gas injected into the anterior chamber was thought to have been directed into the subretinal space by membranes behind the pupillary area. ${ }^{2}$ McDonald et al., made recommendations on injection techniques stressing the importance of achieving a single bubble. They believed that subretinal gas would not complicate the pneumatic retinal procedure if a large gas bubble was present and the retinal breaks were one clock-hour or less in size. ${ }^{3}$ It was implied that large retinal breaks and small gas bubbles were the only prerequisites to this complication.

Gas has been used extensively for the management of superior bullous detachment in the D-ACE technique, ${ }^{5,6}$ for giant retinal tears, ${ }^{7,8}$ and as an internal tamponade following vitrectomy, ${ }^{9}$ without reports of subretinal gas occurring. This suggests that subretinal gas is a complication particular to pneumatic retinopexy.

Two cases of subretinal gas following the surgery for retinal detachment are described. They are important to the understanding of the mechanisms by which gas gains access into the sub-retinal space, they also illustrate the difficulties in management that ensue following this complication.

\section{Case Reports}

\section{Case 1}

A 27-year-old male student presented with a history of floaters and blurred vision in his left eye. Two days previously, he had received a direct blow to his left eye. There was a left posterior vitreous detachment and a superonasal bullous retinal detachment with the macula attached (Fig. 1). 


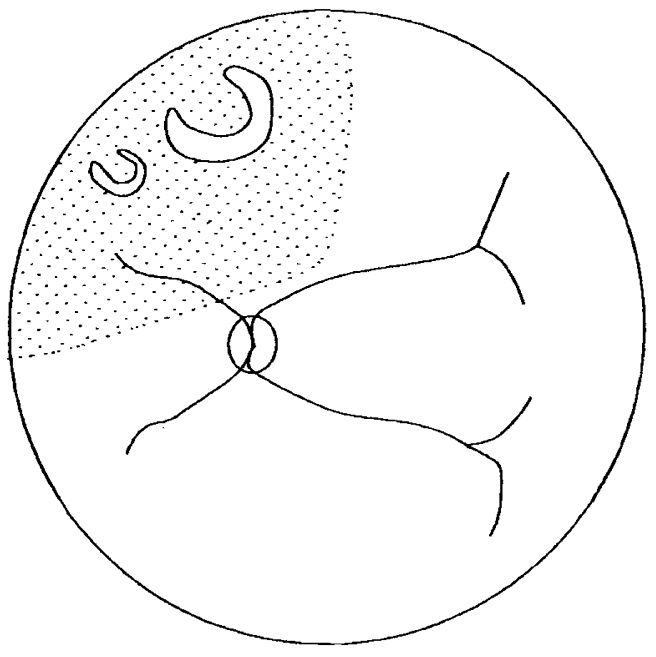

Fig. 1. Retinal diagram of Case 1 with left superonasal retinal detachment and one large and one small horseshoe break.

There were two postequatorial retinal breaks, one of which was large at $11 / 2$ clock hours in size, the other was small. He was treated with drainage of subretinal fluid (SRF) in the superonasal quadrant, and sequential injection of $1 \mathrm{ml}$ of sulphur hexafluoride as a single bubble at the pars plana in the inferotemporal quadrant. The retina was flat after the injection of gas. Cryotherapy was applied but no explant was used. The patient was postured prone with the left cheek resting against a pillow. On the first postoperative day, a large single bubble of gas was seen underneath the retina which took on a grey metallic sheen. Two small bubbles of gas were also seen in the vitreous cavity. The retina was totally detached with the detachment extending anteriorly to involve the non-pigmented epithelium of the pars plana (NPEPP). The two retinal breaks had coalesced to form a single large tear.

\section{Case 2}

A 52-year-old male patient presented with a fiveday history of floaters and an inferonasal visual field defect in the right eye. He was myopic. There was no significant past ophthalmic history. Fundoscopy of the right eye showed a posterior vitreous detachment and a superotemporal retinal detachment with the macula attached. Two equatorial retinal breaks amounting to one clock hour were present in the superotemporal retina (Fig. 2). At surgery, the patient was treated with cryotherapy followed by drainage of SRF at the equator in the upper temporal quadrant, drainage was difficult and only a limited amount of SRF was drained. One millilitre of air was injected at the pars plana temporally and this was complicated by its dispersion into a number of small bubbles. A $7 \mathrm{~mm}$ radial silicone sponge was applied over the two retinal breaks. At the end of the operation, the retinal breaks appeared supported though not closed and there was some residual SRF present around and posterior to the indent. No subretinal gas was seen. The patient was postured prone with the left cheek resting on a pillow. The next day he was found to have a bullous upper half retinal detachment with a single bubble of subretinal air and a smaller air bubble in the vitreous cavity. The retina was deeply detached over the scleral indent, and the two retinal breaks coalesced into one large tear.

\section{Management of Subretinal Gas}

\section{Case 1}

An attempt was made on the first postoperative day to posture the patient to encourage the subretinal gas to pass through the retinal break and into the vitreous cavity. This was unsuccessful, as the buoyant bubble always assumed the uppermost position in the eye (Fig. 3). On the second postoperative day, we proceeded to a pars plana vitrectomy. At operation, several problems were encountered. Firstly, access to the vitreous cavity was difficult. With the patient supine on the operating table, the retina, displaced by the gas bubble, was up against the posterior surface of the crystalline lens obscuring the view of the vitreous cavity. Access into the vitreous cavity was only possible by moving the globe so that the subretinal gas and the detached retina moved away from the crystalline lens. Secondly, an attempt was made to drain the subret-

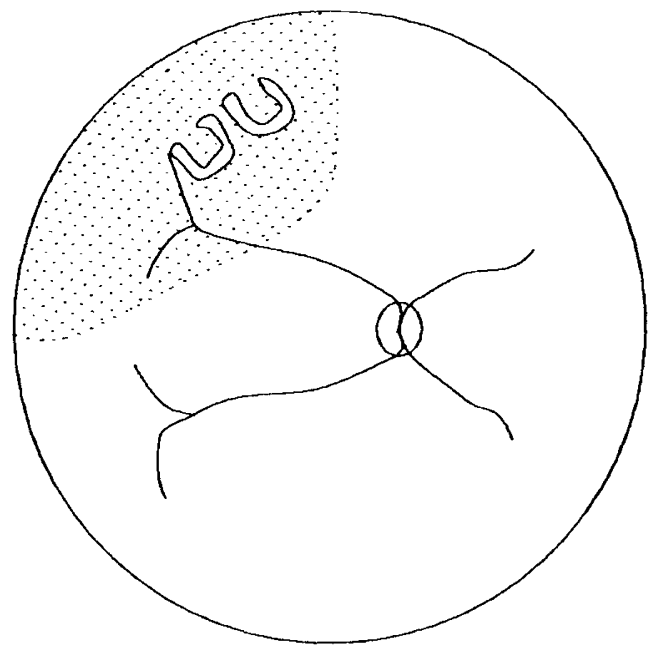

Fig. 2. Retinal diagram of Case 2 with right superotemporal retinal detachment and two horseshoe breaks. 


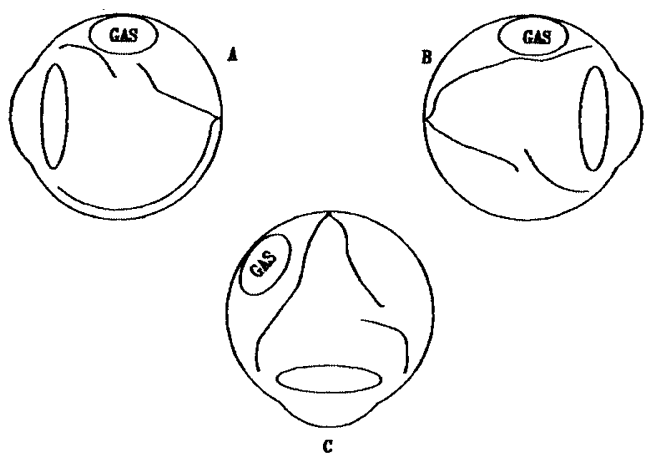

Fig. 3. Effect of posture on position of subretinal gas. A-Subretinal gas occupies the upper most position in the eye, preventing evacuation through break. $B \&$ $C-$ Extension of retinal detachment when posture is changed.

inal gas by a sclerotomy at the pars plana, in the upper temporal quadrant directly over the gas bubble. This resulted in only a small amount of subretinal gas being expelled before the NPEPP and the anterior retina were incarcerated into the sclerotomy preventing further evacuation of the subretinal gas. Thirdly, the vitrectomy was hampered by the mobile retina which often came close to and surrounded the vitreous cutter. After the vitrectomy, fluid in the vitreous cavity was exchanged for air using a drainage needle. The effect of this was the displacement of the SRF and the subretinal bubble of gas posteriorly, until the air in the vitreous cavity came into contact with the subretinal gas through the retinal break. The smaller subretinal gas bubble then emptied into the larger intravitreal bubble of air.

Further cryotherapy and injection of silicone oil resulted in retinal reattachment. The retina remained attached for two weeks and a further operation was performed to remove the silicone oil. Three weeks postoperatively, the patient presented again with a total retinal detachment associated with two small retinal breaks opposite the superotemporal sclerotomy site. This was treated with cryotherapy, the application of a radial sponge explant and nondrainage of SRF. This resulted in retinal reattachment which remained attached at two years.

\section{Case 2}

The air bubble underneath the retina gradually absorbed over three days. The retinal detachment extended to become a total detachment. On the fourth day, a pars plana vitrectomy was performed. SF6/air $(30 \% / 70 \%)$ was exchanged for the preretinal and SRFs. The radial explant was removed, cryotherapy was applied and a circumferential 287 scleral tyre was secured over the large retinal break. The retina became completely reattached for two weeks, but subsequently inferior SRF appeared due to incomplete closure of the superior break, the previous buckle having been placed too posteriorly. At reoperation the eye was rebuckled with an encircling procedure, and the superior buckle augmented by moving the 287 tyre more anteriorly. Further air/SF6 exchange was performed. Following this procedure the retina became completely reattached and has remained so for the last two years.

\section{Discussion}

These two cases provide insight into the mechanism by which gas in the vitreous cavity may gain access into the subretinal space. Two crucial events which preceded the complication of subretinal gas are illustrated in the cases presented. Firstly two retinal breaks were located in close proximity to one another, these breaks were later found to have coalesced into one large tear, providing a large route of access into the subretinal space. Secondly, vitreous traction at the site of the retinal breaks was not relieved in either of the two cases. In Case 1 no scleral buckling procedure was used, and in Case 2, the retinal breaks remained open after scleral buckling.

The two retinal breaks coalescing into a single large tear was the result of the gas bubble forcing its way through one break to enter the subretinal space. This force is provided by the buoyancy of the gas bubble, and its direction is dictated by the posture adopted by the patient. Although the buoyancy would cause the bubble to float upwards, it still has to pass through the retinal break into the subretinal space. In Case 2 incomplete closure of the break with the retina remaining elevated from the retinal pigment epithelium, allowed access into the subretinal space. In Case 1 access into the subretinal space was denied by the drainage of SRF. Some other mechanism must have been responsible in Case 1 to displace the neuroretina from the pigment epithelium. This lifting force on the retina could only have been provided by active vitreoretinal traction. The precise location of the gas bubble is however essential to the understanding of the forces it generates. If the gas bubble was within the vitreous gel, it would act to close the retinal break by approximating the operculum towards the retinal pig- 




Fig. 4. Gas bubble injected into the vitreous gel acts to close breaks.

ment epithelium (Fig. 4). Therefore the gas bubble must either have been injected directly into the retrohyaloid space, or have passed from the gel into the retrohyaloid space through holes in the posterior vitreous face (as in high myopia). In this position, the gas bubble would be channelled towards the posterior border of the vitreous base, that is towards the superiorly located operculum if during the postoperative period the patient assumed an upright posture. This provided an acute static tractional force to the posterior vitreous face and its attachment to the oper-

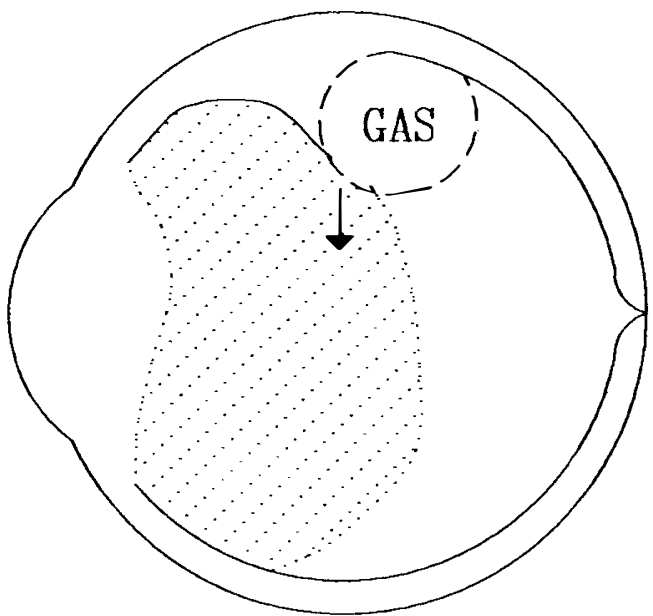

Fig. 5. Gas bubble in the retrohyloid space acts to open breaks, facilitating the passage of gas through the break and into the subretinal space. culum and surrounding retina (Fig. 5). This traction is responsible for lifting the retina and the subsequent passage of retrohyaloid fluid and gas through the break into the subretinal space.

McDonald recommended posturing the patient to evacuate the subretinal gas into the vitreous cavity. ${ }^{3}$ Case 1 shows that posturing is unlikely to evacuate the subretinal gas and may lead to an extension of the retinal detachment and should be avoided.

In conclusion gas in the retrohyloid space may increase traction on the anterior aspect of retinal tears and thereby encourage the passage of gas into the sub-retinal space. Pressure on the breaks ruptured intervening retinal tissue between them so that one large break was formed. Unrelieved vitreoretinal traction or open breaks in the presence of gas in the retrohyaloid space is a requirement for gas to pass through the break and enter the subretinal space. We believe the relief of vitreoretinal traction or the closure of the retinal breaks by a scleral explant would prevent the passage of gas bubbles underneath the retina.

\section{References}

${ }^{1}$ Chawla $\mathrm{HB}$ and Birchall $\mathrm{CH}$ : Intravitreal air in retinal detachment surgery. Br J Ophthalmol 1973, 57: 60-70.

${ }^{2}$ Lincoff $\mathrm{H}$, Kreissig I, Jakobiec F: The inadvertent injection of gas beneath the retina in a pseudophakic eye. Ophthalmology 1986, 93: 408-10.

${ }^{3}$ McDonald RH, Abrams GW, Irvine AR et al: The management of subretinal gas following attempted pneumatic retinal reattachment. Ophthalmology 1987, 94 319-26.

${ }^{4}$ Lowe MA, McDonald R, Campo RV, Boyer DS et al: Pneumatic retinopexy: Surgical results. Arch Ophthalmol 1988, 106: 1672-6.

${ }^{5}$ Gilbert C and McLeod D: D-ACE surgical sequence for selected bullous retinal detachments. $\mathrm{Br} J$ Ophthalmol 1985, 69: 733-6.

${ }^{6}$ Stanford MR and Chignell AH: Surgical treatment of superior bullous rhegmatogenous retinal detachments. Br J Ophthalmol 1985, 69: 729-32.

${ }^{7}$ Vidaurri-Leal J, De Bustros S, Michels RG: Surgical treatment of Giant retinal tears with inverted posterior retinal flaps. Am J Ophthalmol 1984, 98: 463-6.

${ }^{8}$ Machemer R and Allen AW: Retinal tears 180 degrees and greater. Management with vitrectomy and intravitreal gas. Arch Ophthalmol 1976, 94: $12-40$.

${ }^{9}$ Freeman HK and Castillejos ME: Current management of giant retinal tears: Results with vitrectomy and total air-fluid exchange in 95 cases. Trans Am Ophthalmol Soc 1981, 179: 89-102. 